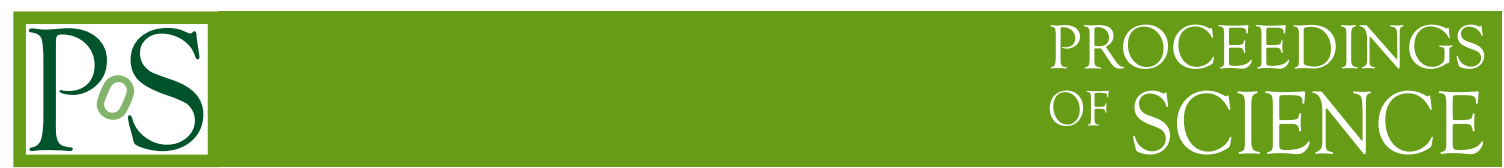

\title{
BSM reach of single top-quark measurements
}

\author{
Alberto Tonero ${ }^{a, *}$ \\ ${ }^{a}$ Ottawa-Carleton, Institute for Physics, Carleton University \\ 1125 Colonel By Drive, Ottawa, ON, K1S 5B6, Canada \\ E-mail: alberto.tonero@gmail.com
}

Overview about the reach of BSM physics using single top production at the LHC.

The Ninth Annual Conference on Large Hadron Collider Physics - LHCP2021

7-12 June 2021

Online

${ }^{*}$ Speaker 


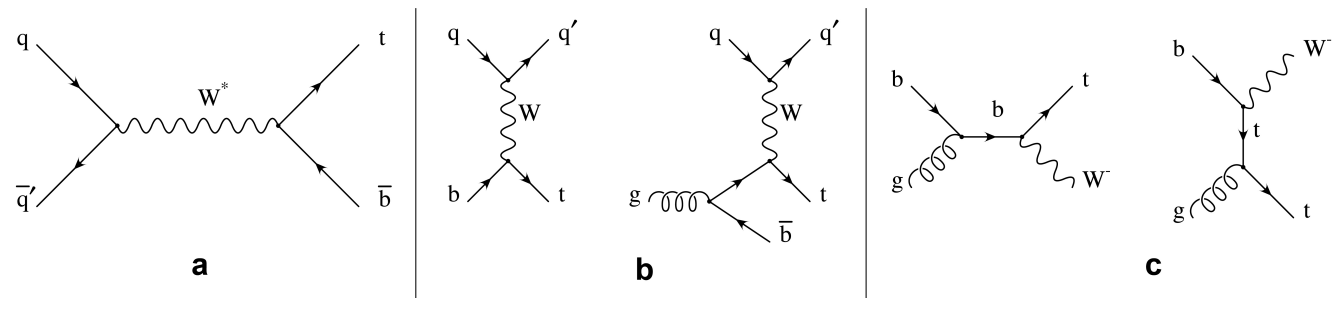

Figure 1: The three main production channels of single top: a) $s$-channel, b) $t$-channel and c) $t W$-channel.

\section{Introduction}

Despite the numerous successes of the Standard Model (SM), there exist phenomena like Neutrino masses, Baryon Asymmetry of the Universe, Dark Matter, hierarchy problem, flavor, etc... which require the presence of Beyond the Standard Model (BSM) physics in order to be explained. Due to its high mass, the top-quark is believed to represent a unique portal to New Physics (NP). At the Large Hadron Collider (LHC), the physics of the top quark entered the era of precision measurements due to the large number of top particles produced in proton collisions at $13 \mathrm{TeV}$. Heavy New Physics may manifest itself in the form of effective couplings involving the top-quark (Wtb-vertex, four fermion interactions, etc...) that can be probed in single-top production. There are three main channels for single top production which are characterized by different virtualities of the $W$-boson: $s$-, $t$ - and $t W$-channel. The tree level diagrams for these three production channels are shown in Figure 1. We have:

a) s-channel $q_{W}^{2}>\left(m_{t}+m_{b}\right)^{2}$

b) $t$-channel $q_{W}^{2}<0$

c) $t W$-channel $q_{W}^{2}=m_{W}^{2}$

The $13 \mathrm{TeV}$ cross sections are [1]: $\sigma_{t}(p p \rightarrow t j+\bar{t} j) \simeq 136+82 \mathrm{pb}$ aNNLO QCD, $\sigma_{t W}(p p \rightarrow$ $t W+\bar{t} W) \simeq 70 \mathrm{pb}$ aNNLO QCD, $\sigma_{s}(p p \rightarrow t b+\bar{t} b) \simeq 7+4 \mathrm{pb} @ 13 \mathrm{TeV}$ aNNLO QCD. Single-top $t$-channel is the dominant process.

\section{Wtb anomalous couplings}

Heavy BSM physics can affect the top-quark weak gauge interactions and manifest in the form of effective couplings at low energies. The most general Lorentz-invariant vertex is parametrized as [2-4]:

$$
\mathcal{L}_{W t b}=-\frac{g}{\sqrt{2}} \bar{b} \gamma^{\mu}\left(V_{L} P_{L}+V_{R} P_{R}\right) t W_{\mu}^{-}-\frac{g}{\sqrt{2}} \bar{b} \frac{i \sigma^{\mu v} q_{v}}{m_{W}}\left(g_{L} P_{L}+g_{R} P_{R}\right) t W_{\mu}^{-}+\text {h.c. }
$$

In the SM at tree level we have $V_{L}=V_{t b} \simeq 1$ and $V_{R}=g_{L}=g_{R}=0$. BSM physics can provide additional contributions to $V_{L, R}$ and $g_{L, R}$ as shown in [5-12]. 


\subsection{Anomalous couplings contributions to observables}

Using the effective vertex in eq. (1), it is possible to compute the $s-, t$ - and $t W$-channel cross sections, which can be parametrized as follows

$$
\sigma_{i}=\sigma_{i}^{\mathrm{SM}}+K_{i} \Delta \sigma_{i}
$$

where $K_{i}$ are the k-factors and $\Delta \sigma_{i}$ are the LO NP contributions, computed in the limit $m_{b}=0$ [11]:

$$
\begin{aligned}
\Delta \sigma_{t} & =a_{0} x_{0}+a_{m} x_{m}+a_{p} x_{p}+a_{5} x_{5} \quad \Delta \sigma_{s}=b_{0} x_{0}+b_{m} x_{m}+b_{p} x_{p}+b_{5} x_{5} \\
\Delta \sigma_{t W} & =c_{0} x_{0}+c_{m} x_{m}+c_{p} x_{p}+c_{5} x_{5}
\end{aligned}
$$

with

$$
x_{0}=\left|V_{L}+\frac{m_{W}}{m_{t}} g_{R}\right|^{2}+\left|V_{R}+\frac{m_{W}}{m_{t}} g_{L}\right|^{2}-1 \quad x_{5}=\frac{m_{t}^{2}}{m_{W}^{2}}\left(\left|g_{L}\right|^{2}+\left|g_{R}\right|^{2}\right)
$$

and

$$
x_{m}=\left|V_{L}+\frac{m_{t}}{m_{W}} g_{R}\right|^{2}-1 \quad x_{p}=\left|V_{R}+\frac{m_{t}}{m_{W}} g_{L}\right|^{2}
$$

The anomalous couplings contribute also to helicity $(*)$, normal $(N)$ and transverse $(T)$ polarization fractions [13] which are defined as:

$$
\frac{1}{\Gamma} \frac{d \Gamma}{d \theta_{l}^{x}}=\frac{3}{8}\left(1+\cos \theta_{l}^{x}\right)^{2} F_{R}^{x}+\frac{3}{8}\left(1-\cos \theta_{l}^{x}\right)^{2} F_{L}^{x}+\frac{3}{4} \sin ^{2} \theta_{l}^{x} F_{0}^{x}
$$

At LO, in the limit $m_{b}=0$, the expressions for $F_{i}^{*}$ can be found here [11]. Other powerful observables to probe anomalous couplings are Forward-Backward Asymmetries ( $P$ top polarization) [13] constructed in terms of polarization fractions

$$
A_{\mathrm{FB}}=\frac{3}{4}\left[F_{R}^{*}-F_{L}^{*}\right] \quad A_{\mathrm{FB}}^{N, T}=\frac{3}{4} P\left[F_{R}^{N, T}-F_{L}^{N, T}\right]
$$

\subsection{Current and expected bounds}

95\% CL limits on anomalous couplings obtained by combining helicity fractions, single top production cross section and $A_{\mathrm{FB}}$ asymmetries can be found in $[14,15]$. Those limits are summarized in Table 1 where $\operatorname{Im} V_{L}$ is set to be 0 .

\begin{tabular}{ccccc}
\hline \hline & $V_{L}$ & $V_{R}$ & $g_{L}$ & $g_{R}$ \\
\hline $\operatorname{Re}$ & {$[0.85,1.08]$} & {$[-0.35,0.39]$} & {$[-0.23,0.22]$} & {$[-0.07,0.07]$} \\
$\operatorname{Im}$ & - & {$[-0.37,0.39]$} & {$[-0.22,0.23]$} & {$[-0.20,0.15]$} \\
\hline $\operatorname{Re}$ & 1 & {$[-0.28,0.32]$} & {$[-0.17,0.19]$} & {$[-0.05,0.02]$} \\
$\operatorname{Im}$ & - & {$[-0.30,0.30]$} & {$[-0.19,0.18]$} & {$[-0.11,0.10]$} \\
\hline
\end{tabular}

Table 1: The first two lines show 95\% CL limits on anomalous couplings allowing all couplings to vary simultaneously, these limits are extracted from collected data at LHC and Tevatron [14]. The bottom two lines show limits where $\operatorname{Re} V_{L}$ is kept fixed to 1 and include the extrapolated results from the HL-LHC (3000 $\left.\mathrm{fb}^{-1}\right)[15]$. 


\section{Constraining NP with $p p \rightarrow t l v$}

Differential distributions in $p p \rightarrow t l v \rightarrow b l v l v$ [16] can be used to constrain EFT four fermion operators involving heavy quarks and leptons

$$
\begin{gathered}
\frac{c_{Q l_{i}}^{3(1)}\left(\bar{l}_{i L} \gamma^{\mu} \sigma^{I} l_{i L}\right)\left(\bar{Q}_{L} \gamma_{\mu} \sigma_{I} Q_{L}\right)}{\Lambda^{2}} \quad \frac{c_{t l_{i}}^{S(1)}\left(\bar{l}_{i L} e_{i R}\right) \epsilon\left(\bar{Q}_{L} t_{R}\right)+\text { h.c. }}{\Lambda^{2}} \\
\frac{c_{t l_{i}}^{T(1)}\left(\bar{l}_{i L} \sigma^{\mu v} e_{i R}\right) \epsilon\left(\bar{Q}_{L} \sigma_{\mu \nu} t_{R}\right)+\text { h.c. }}{\Lambda^{2}}
\end{gathered}
$$

A sequential $W^{\prime}$ model with coupling $k_{L}$ and mass $M_{W^{\prime}}$

$$
\mathcal{L}=-\frac{g_{W}}{\sqrt{2}} k_{L} \bar{v}_{L} \gamma^{\mu} e_{L} W_{\mu}^{\prime}-\frac{g_{W}}{\sqrt{2}} k_{L} \bar{t}_{L} \gamma^{\mu} b_{L} W_{\mu}^{\prime}+\text { h.c. }
$$

can be matched to the EFT operators as follows:

$$
C_{2} \equiv \frac{c_{Q l}^{3(1)}}{\Lambda^{2}}= \pm \frac{g_{W}^{2} k_{L}^{2}}{4 M_{W^{\prime}}^{2}} \quad \frac{c_{t l}^{S(1)}}{\Lambda^{2}}=\frac{c_{t l}^{T(1)}}{\Lambda^{2}}=0
$$

Current and expected bounds on the sequential $W^{\prime}$ model with comparison are obtained in [17]

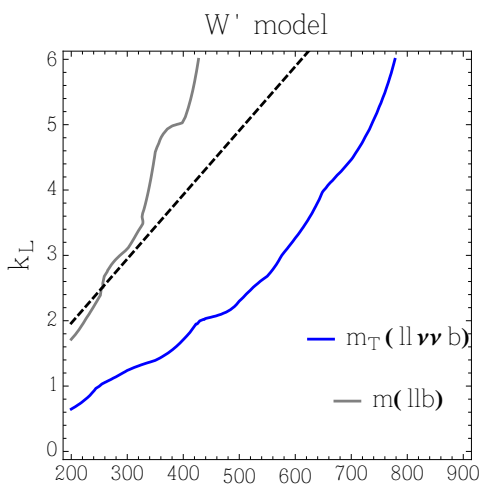

$M[\mathrm{GeV}]$

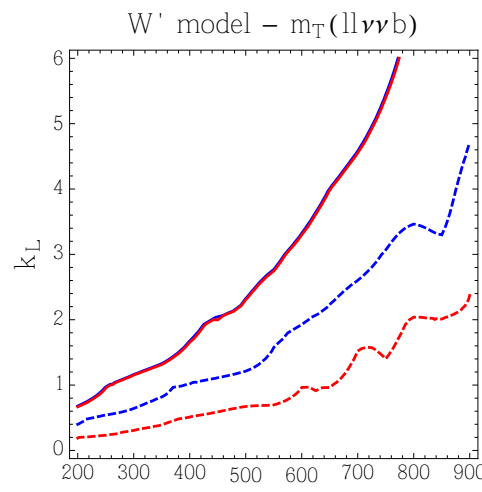

$M[\mathrm{GeV}]$

Figure 2: Left: current bounds using differential distributions at $36.1 \mathrm{fb}^{-1}$. Right: expected bounds at 300 $\mathrm{fb}^{-1}$ (blue) and $3000 \mathrm{fb}^{-1}$ (red).

\section{Conclusion}

LHC is a top factory which allows to study top-quark properties very precisely. Single top is a great probe of NP because its production mechanisms have cross sections directly proportional to the $W t b$ vertex. Heavy BSM physics may manifest at low energy by modifying the $W t b$ vertex. Current measurements constrain $W t b$ anomalous couplings at the 0.1 level, future sensitivity may reach 0.01 level. EFT limits imply $\Lambda \gtrsim 1 \mathrm{TeV}$. Less constrained SMEFT operators may be probed in differential measurements of the full final state $p p \rightarrow t l v$. 


\section{References}

[1] N. Kidonakis, PoS DIS2015, 170 (2015) doi:10.22323/1.247.0170 [arXiv:1506.04072 [hep$\mathrm{ph}]]$.

[2] C. S. Li, R. J. Oakes and T. C. Yuan, Phys. Rev. D 43, 3759-3762 (1991) doi:10.1103/PhysRevD.43.3759

[3] G. L. Kane, G. A. Ladinsky and C. P. Yuan, Phys. Rev. D 45, 124-141 (1992) doi:10.1103/PhysRevD.45.124

[4] F. del Aguila and J. A. Aguilar-Saavedra, Phys. Rev. D 67, 014009 (2003) doi:10.1103/PhysRevD.67.014009

[5] D. O. Carlson, E. Malkawi and C. P. Yuan, Phys. Lett. B 337, 145-151 (1994) doi:10.1016/0370-2693(94)91459-1

[6] D. Atwood, S. Bar-Shalom, G. Eilam and A. Soni, Phys. Rev. D 54, 5412-5416 (1996) doi:10.1103/PhysRevD.54.5412 [arXiv:hep-ph/9605345 [hep-ph]].

[7] E. H. Simmons, Phys. Rev. D 55, 5494-5500 (1997) doi:10.1103/PhysRevD.55.5494 [arXiv:hep-ph/9612402 [hep-ph]].

[8] M. Beneke, I. Efthymiopoulos, M. L. Mangano, J. Womersley, A. Ahmadov, G. Azuelos, U. Baur, A. Belyaev, E. L. Berger and W. Bernreuther, et al. [arXiv:hep-ph/0003033 [hep$\mathrm{ph}]]$.

[9] J. A. Aguilar-Saavedra, Nucl. Phys. B 812, 181-204 (2009) doi:10.1016/j.nuclphysb.2008.12.012 [arXiv:0811.3842 [hep-ph]].

[10] M. Fabbrichesi, M. Pinamonti and A. Tonero, Eur. Phys. J. C 74, no.12, 3193 (2014) doi:10.1140/epjc/s10052-014-3193-8 [arXiv:1406.5393 [hep-ph]].

[11] Q. H. Cao, B. Yan, J. H. Yu and C. Zhang, Chin. Phys. C 41, no.6, 063101 (2017) doi:10.1088/1674-1137/41/6/063101 [arXiv:1504.03785 [hep-ph]].

[12] A. Arhrib and A. Jueid, JHEP 08, 082 (2016) doi:10.1007/JHEP08(2016)082 [arXiv:1606.05270 [hep-ph]].

[13] J. A. Aguilar-Saavedra and J. Bernabeu, Nucl. Phys. B 840, 349-378 (2010) doi:10.1016/j.nuclphysb.2010.07.012 [arXiv:1005.5382 [hep-ph]].

[14] F. Déliot, R. Faria, M. C. N. Fiolhais, P. Lagarelhos, A. Onofre, C. M. Pease and A. Vasconcelos, Phys. Rev. D 97, no.1, 013007 (2018) doi:10.1103/PhysRevD.97.013007 [arXiv:1711.04847 [hep-ph]].

[15] F. Déliot, M. C. N. Fiolhais and A. Onofre, Mod. Phys. Lett. A 34, no.18, 1950142 (2019) doi:10.1142/S0217732319501426 
[16] M. Aaboud et al. [ATLAS], Eur. Phys. J. C 78, no.3, 186 (2018) doi:10.1140/epjc/s10052018-5649-8 [arXiv:1712.01602 [hep-ex]].

[17] D. Stolarski and A. Tonero, JHEP 08, no.08, 036 (2020) doi:10.1007/JHEP08(2020)036 [arXiv:2004.07856 [hep-ph]]. 